CARNETS OE Carnets de géographes

GÉOGRAPHES.

\title{
Quatre géographes face à leurs émotions
}

Retour réflexif face à l'expérience de l'événement séisme du 25 avril 2015 au Népal

Véronique André-Lamat, Marie Faulon, Etienne Jacquemet et Isabelle Sacareau

\section{(2) OpenEdition}

Journals

Édition électronique

URL : http://journals.openedition.org/cdg/665

DOI : $10.4000 /$ cdg. 665

ISSN : 2107-7266

Éditeur

UMR 245 - CESSMA

Référence électronique

Véronique André-Lamat, Marie Faulon, Etienne Jacquemet et Isabelle Sacareau, «Quatre géographes face à leurs émotions », Carnets de géographes [En ligne], 9 | 2016, mis en ligne le 30 novembre 2016, consulté le 30 avril 2019. URL : http://journals.openedition.org/cdg/665 ; DOI : 10.4000/cdg.665

La revue Carnets de géographes est mise à disposition selon les termes de la Licence Creative Commons Attribution - Pas d'Utilisation Commerciale - Pas de Modification 4.0 International. 


\title{
QUATRE GEOGRAPHES FACE A LEURS EMOTIONS \\ Retour réflexif face à l'expérience de l'événement séisme du 25 avril 2015 au Népal
}

\author{
Véronique ANDRE-LAMAT (VAL) \\ Laboratoire PASSAGES UMR 5319 \\ Université Bordeaux Montaigne - Géographie \\ Veronique.andre-lamat@cnrs.fr \\ Marie FAULON (MF) \\ LABORATOIRE PASSAGES UMR 5319 \\ Université Bordeaux Montaigne - Géographie \\ Marie.faulon@gmail.com \\ Etienne JACQUEMET (EJ) \\ Laboratoire PASSAGES UMR 5319 \\ Université Bordeaux Montaigne - Géographie \\ Etienne.jacquemet@cnrs.fr \\ Isabelle SACAREAU (IS) \\ Laboratoire PASSAGES UMR 5319 \\ Université Bordeaux Montaigne - Géographie \\ isabelle.sacareau@cnrs.fr
}

\begin{abstract}
Résumé
A partir de témoignages relatifs au séisme qui a touché le Népal le 25 avril 2015, cet article s'interroge sur la façon dont quatre chercheurs ont géré cette catastrophe à travers le prisme de leurs émotions, en la vivant directement sur place ou à distance. La lecture de ces différentes expériences montre que l'emplacement des différents chercheurs interfère peu dans la nature et l'organisation des émotions, dans la mesure où celles-ci se révèlent assez similaires. Les mécanismes à l'origine d'états affectifs intenses et leurs effets différenciés sur les individus se fondent pour une grande part sur des référentialités personnelles ayant trait à des évènements passés, au terrain ou aux statuts et carrières des chercheurs eux-mêmes. Mais, les émotions sont aussi canalisées plus ou moins fortement en fonction de la capacité de chacun à agir et à s'accommoder de l'espace et de la distance.
\end{abstract}

Mots-clefs : émotions, séisme, risques, Népal, recherche de terrain 


\begin{abstract}
Within the context of the April 25, 2015 Nepal earthquake, this paper examines how four researchers deal with the disaster throught the lens of their emotions whether they are on site or away. Their personnal accounts show that their localisation both in Nepal and in France does not interfere much in the nature and the organisation of emotions. Those are indeed almost the same. Aside from an extra-ordinary event, the mechanisms which originally produce emotions and their differential impact on individuals, are widely based on various and personal references related to the researchers past experiences, fieldwork, careers and status. Finally, these emotions are also channeled more or less strongly according to each person's capacity to act and adapt him or herself to space and distance.
\end{abstract}

Keywords: emotions, earthquake, risk, Nepal, field research 


\section{Introduction}

25 avril 2015. Le tremblement de terre attendu depuis des décennies au Népal a lieu. Les membres de l'équipe PRESHINE ${ }^{1}$ basculent dans l'expérience brutale du séisme : certains la vivent sur place, d'autres à distance, mais tous la ressentent dans leur corps. L'équipe a alors sur le terrain - la région de l'Everest - un doctorant, deux stagiaires et trois chercheurs. L'expérience de cet évènement engendre une vive émotion au sein du collectif. Elle place les chercheurs dans "une situation d'alerte et d'urgence" (Charon et al., 2014, p. 96), une situation extraordinaire, en " rupture de continuité » (Bloch \& Vurpillot, 1994, p. 262 ; Rimé, 2005 , p. 52). Face à la catastrophe, le bouleversement psychique, plus ou moins conscient, long, intense ou invasif, s'accompagne de réactions stimulantes et/ou désorganisatrices qui interfèrent non seulement avec l'ensemble des attitudes et perspectives des individus (Sillamy, 1989), mais aussi - c'est la thèse défendue dans cet article - sur leurs propres références spatiales et temporelles.

Ainsi, ce carnet de terrain, écrit par quatre chercheurs du programme, vise-t-il d'abord à tenter de porter un regard réflexif sur les différents types d'états affectifs générés par la perturbation de leur environnement de vie et de travail. La notion d'états affectifs recouvre dans cet article tout à la fois les émotions et les sentiments. Selon Charon et al., (2014), les sentiments résultent d'expériences subjectives et cognitives. Ils sont plus durables, moins intenses et invasifs que les émotions. Toutefois, émotions et sentiments servent les mêmes objectifs, ils amènent les individus à réagir en fonction de besoins. Certains états affectifs désignés ci-après sous le terme de sentiments pourront donc être considérés comme des émotions. Il s'agit ensuite de comprendre comment, au cours et durant les jours qui ont suivi le séisme, ces états ont redéfini, dans un rapport d'interspatialité, la perception et la relation que ces chercheurs ont entretenues avec l'espace, la distance et leur terrain de recherche.

Dans une première partie, chaque auteur livre son témoignage sur son vécu de l'événement. Cette restitution, en partie reconstruite - puisque écrite par trois des auteurs a posteriori constitue le matériau de première main de cette analyse qui interroge la façon dont se déclenchent les émotions. Ces récits révèlent pour tous, des états affectifs communs de nature similaire. Toutefois, une deuxième partie montre que la relation affective au terrain et les trajectoires individuelles sont des dimensions essentielles à prendre en compte pour comprendre les ressorts du déclenchement des émotions et leur intensité. La troisième partie s'intéresse à la manière donc chacun a géré les émotions produites par la catastrophe et questionné sa capacité à maîtriser et à appréhender l'espace et la distance, à travers ce que Michel Lussault appelle la compétence d'arrangement et de placement des individus: " cette capacité [qui] consiste à savoir trouver pour soi, les autres, les objets, la bonne place » (2009: 84).

\section{Népal - France : un événement, des émotions en partage}

L'objectif de cette première partie est de révéler la portée émotionnelle de l'événement séisme pour quatre membres de l'équipe PRESHINE en décrivant la façon dont il a été

1 ANR-13-SENV-0005, Pressions sur la ressources en eau et en sols dans l'Himalaya népalais, programme de recherche pluridisciplinaire coordonné par I. Sacareau (UMR 5185 ADESS-PASSAGES). 
ressenti sur le moment et dans les jours qui l'ont suivi. Quels types d'émotions sont alors apparus ou non pour chacun ? À quels moments?

\section{EJ : Au cœur de l'évènement (extrait carnet de terrain)}

D'un coup Alix se retourne : " un tremblement de terre!" crie-t-elle. Je sens à mon tour le sol se dérober sous mes pieds [...] Un bruit effrayant se produit au-dessus, en dessous ou tout autour de nous. J'ai l'impression que la montagne s'effondre. Qu'elle va nous tomber directement dessus [...]. Je me mets à courir à travers la forêt mais suis persuadé que nous sommes pris au piège, que nous allons mourir. [...] Au bout de trente secondes (peut-être) je retombe sur le sentier, la secousse s'est arrêtée. [...] Remis de nos émotions, nous décidons de remonter à Tengboche, Harka et Céline nous rejoignent. À Tengboche on se sent en sécurité. [Mais] nous pensons rapidement à tous les touristes que nous avons croisés plus tôt en descendant la gorge, près du pont. $Y$-a-t-il des victimes ? Où se trouvait l'épicentre ? Est-ce que le barrage devant le lac Imja a tenu ? [...] La neige et le brouillard ajoutent à l'angoisse et à la confusion. [...] Et si le tremblement de terre avait touché l'ensemble du Népal ? [...] Où se trouvent Sonam, Lhakpa et tous les autres ? Les filles envoient immédiatement des messages à leurs familles : "si vous entendez parler d'un séisme au Népal, on va bien ". Moi je n'ai plus de batteries. [...] Je demande à allumer la radio mais rien n'émet. [...] Puis les premières nouvelles de l'extérieur commencent à arriver. Par téléphone satellite, une guide suisse dit qu'on parle déjà du tremblement en boucle sur CNN et que "l'Europe est au courant ". On dit que c'est un très gros séisme de magnitude 7.7, qu'il a frappé à $70 \mathrm{~km}$ de Katmandou et que la grande tour Dhardhara s'est écroulée. Quelque part c'est assez positif, car le message ne dit pas non plus que Katmandou est complètement détruit. N'empêche, très vite on réalise. [...] Et puis très vite on sent venir de gros problèmes: plus de réseau téléphonique, de banques ou d'aéroport, comment va-t-on faire ? Et que va-t-on faire ? Et ma thèse ?! [...] Nous ne recevons qu'en fin d'après-midi les premiers messages de France " grosse secousse à Katmandou, 7.9 entre Pokhara et Katmandou, donnez des nouvelles vite ". Malgré les discussions et les rires qui reprennent dans le lodge, on a un peu envie de pleurer pour les Népalais. J'arrête mes notes sur un message aussi rassurant que déprimant: "J'ai eu les parents d'Etienne. C'est un très gros séisme et le bilan sera lourd surtout à Katmandou ».

\section{IS : Elargissement spatial et individualisation de l'émotion}

25 avril 2015. La nouvelle me cueille au saut du lit en allumant la radio. Le Népal et ses lieux qui me sont familiers font brutalement irruption dans mon espace/temps quotidien par l'intermédiaire de la radio et d'un événement qui pourtant ne me surprend pas. Le Népal a déjà connu des séismes. Bien que ma compassion soit immédiate pour ce pays que j'aime, j'ai d'abord tendance à minimiser l'événement. Cependant, très vite, il me faut savoir où la catastrophe s'est déroulée. Quels lieux ont été touchés ? Y-a-t-il des victimes ? La question du " où? " l'emporte sur toutes les autres, car j'ai de nombreux amis népalais sur place, ainsi que mon doctorant et des membres du programme de recherche dont je suis responsable. À mesure que les informations et les images me parviennent, l'effondrement et l'inquiétude me gagnent: le cœur historique de Katmandou est dévasté, une gigantesque avalanche s'est produite au camp de base de l'Everest: Où est EJ ? Un sentiment d'irréalité me saisit accompagné d'une angoisse diffuse et d'un sentiment d'impuissance, car immobilisée chez moi par un arrêt maladie je ne peux agir concrètement. Soulagement lorsque nous apprenons en fin d'après-midi qu'il est sain et sauf, ainsi que tous les membres de l'équipe 
qui étaient sur le terrain. Décision est rapidement prise avec VAL de faire rapatrier EJ. Tant pis pour la thèse! Que vaut-elle face aux risques à venir, physiques et psychologiques ? Quel sens éthique $y$-a-t-il à enquêter dans un pays dévasté où les gens comptent leurs morts ? Je suis à distance, grâce à VAL, les péripéties de ce rapatriement. Nos longs échanges téléphoniques nous permettent d'exprimer et partager nos inquiétudes, notre colère face aux réactions de certains de nos interlocuteurs. Mais pour moi, le flot des émotions n'est pas encore passé. Les sismologues localisent l'épicentre du séisme dans le district de Gorkha, mon ancien terrain de thèse. Que sont devenus les villageois ? J'ai tellement conscience de leur vulnérabilité face à une catastrophe d'une telle ampleur. Et puis c'est la terrible nouvelle que Dile, notre informateur de terrain, accompagnait ce groupe de Français, disparus dans une avalanche au Langtang dont tous les médias parlent. Comment vont réagir nos jeunes qui ont partagé une grande partie de leur terrain avec lui et les protéger de cette émotion là ? Car je sais au fond de moi qu'il ne reviendra pas. Mais je ne peux pas pleurer. Pas question de me laisser submerger par mon émotion ou celle des autres. Je me précipite fébrilement dans la recherche d'information afin de servir de relai entre mes collègues chercheurs, les associations françaises de solidarité que je connais et le Népal. C'est par internet et par les réseaux sociaux que je vais désormais suivre l'événement jour après jour jusqu'au rapatriement d'EJ et encore après. Je vis dans une sorte d'apesanteur, dans un temps qui semble se dilater et dans un espace confiné, celui de ma maison et de mon jardin, seul contact avec la matérialité du monde extérieur, car je vis parallèlement au Népal par écran interposé : celui de mon ordinateur, de la télévision et de mon téléphone portable. Ils sont la caisse de résonance d'une émotion mondialisée à laquelle je participe.

Sireuil, 21/12/2015.

\section{VAL : Mettre à distance l'émotion pour y faire face}

25 avril 2015, 11h: "il y a eu un séisme au Népal ». Sidérée, j'intègre l'information en allumant la télévision et mon ordinateur. Des images de Katmandou défilent, la magnitude du séisme est donnée et l'étau de l'angoisse se serre : "ça recommence " et déjà je refoule mes peurs, ces angoisses qui m'accompagnent et avec lesquelles je compose depuis 1996 et mes événements guinéens. J'ouvre le mail d'un glaciologue sur le terrain qui a réchappé au séisme in extremis alors qu'il traversait un lac gelé. Je le lis comme une histoire héroïque, mais en fait elle ne m'intéresse pas. Rien ne m'intéresse d'ailleurs, ni la destruction du cœur historique de Katmandou, ni le nombre de morts, je ne cherche de nouvelles que d'une seule personne. II n'y en a pas. Je téléphone sur son portable népalais: messagerie. J'envoie un mail. J'envoie un texto. J'attends. Entourée mais seule. Je marche. Je réfléchis à la seule question qui m'obnubile : où est-il ?

12h04, mail du glaciologue. Il l'a vu hier avec les 2 stagiaires : "le séisme a eu lieu à 12h00, ils étaient sûrement en train de marcher... donc peu exposés j'espère ". J'ai donc une idée approximative du où. Il faut qu'il soit vivant. Pour moi. Egoïstement, je sais déjà que s'il est mort, je n'y arriverai plus : trop de violence émotionnelle dans mes terrains.

$13 \mathrm{~h} 24$ : il est à Tengboche, vivant, secoué, avec les filles. J'ai l'impression d'avoir gagné un combat. Je reste hors du monde qui m'entoure: les informations en boucle égrènent des bilans provisoires, les morts s'accumulent, les images montrent. Fondamentalement, cela me touche peu, j'y penserai plus tard, car maintenant que je sais l'essentiel, je sais ce qu'il faut que je fasse. Mon évidence est qu'il ne doit pas avoir à décider quoi faire : il faut qu'il rentre, 
il faut qu'il soigne son âme. J'ai désormais un but, essentiel, qui me permettra de ne pas penser aux autres, à Dile mon guide et assistant, de garder à distance toute émotion. Le temps passe, le rapatriement s'avère être une course d'obstacles. Les cartes topographiques et les images satellites mises en ligne me projettent vers les lieux que je connais, que je visualise et évalue dans le contexte post-séisme, en tant que repères spatiaux et points d'appui pour cette opération. J'échange en permanence avec mon directeur de laboratoire, IS et $\mathrm{MF}$, les parents de ceux bloqués sur place : j'agis, j'informe, je rassure. Le $1^{\text {er }}$ mai, je libère ma colère face à la discontinuité du suivi du dossier de rapatriement côté assurance et à ce que j'estime être de l'incompétence. Mais ma colère est plus vaste, plus globale : je suis en colère contre l'événement lui-même, contre la disparition de Dile. Colère bien vaine. EJ rentre et premier soulagement de le savoir les pieds posés sur le sol français. Le corps de Dile est retrouvé au Langtang. Je m'autorise alors à regarder les images de la coulée meurtrière. Aucune chance pour personne. Je pleure, sa mort et le miracle que son corps ait été retrouvé.

Pessac, 21/12/2015.

\section{MF : Ecrire pour mettre à distance}

Cela fait quatre jours que je suis rentrée du Népal. Le retour à ma vie est difficile, j'ai l'impression d'évoluer dans une bulle ouatée, la mise à distance de cette parenthèse népalaise commence à s'opérer. A la veille de cette catastrophe, je regarde mon avenir immédiat, l'écriture de mon mémoire.

Vers 11h, un sms arrive, il y a eu un séisme au Népal. J'éteins mon téléphone, je ne veux pas savoir, je ne veux pas repartir, je veux écrire et en finir. Le lendemain, le temps s'accélère. Je suis submergée d'appels et d'emails de proches qui ne savent pas si je suis rentrée. Je pense à mes collègues sur le terrain. J'envoie un email, je n'imagine pas que quelque chose ait pu mal tourner pour ceux que je connais là-bas. J'y pense sans y penser mais je suis envahie d'informations à mon insu, je n'échappe pas à la vague médiatique qui déferle à la radio et sur les réseaux sociaux sur les destructions, les morts et les miracles. Mon mémoire reste toujours présent dans mon esprit, comme un fil rouge tout au long de l'après-catastrophe, une sorte de rempart intellectuel contre quelque chose dont je n'ai pas la maîtrise. Une question est redondante : comment écrire sur ce terrain que j'ai construit et qui n'existe peutêtre plus ? Je n'arrive pas à en parler, je n'ai qu'un seul interlocuteur, VAL, je ne parle qu'à elle, et les seules questions que je me pose tournent autour de l'écriture du mémoire. Rien d'autre, je ne peux pas. Fortuitement, j'apprends par un article que Dile Gurung, notre guide était l'accompagnateur d'un groupe porté disparu. J'appelle Véronique, je ne veux pas y croire, ce n'est pas possible, pas Dile. Il me ramène sur le terrain, de force. Porté disparu pendant dix jours, je passe mon temps à actualiser le site du Monde et à rebondir vers tous les liens qui pourraient satisfaire ma soif d'informations. Je sens bien que finalement, je n'ai pas réussi à rentrer chez moi. Les souvenirs de notre travail ensemble affluent et me projettent dans un autre espace mais aussi dans le passé et l'écriture reste tout à fait hors de ma portée. Dix jours après, la mort de Dile m'est enfin annoncée, elle personnifie la catastrophe, l'intègre dans mon monde, l'inscrit dans mon corps. Cette victime résume toutes les autres. L'angoissante question de savoir comment écrire, au présent, au passé demeure. II faut que j'évacue la présence de Dile dans mon esprit. Je m'enferme et j'écris un préambule, que faire d'autre ? J'écris et je mets à distance.

Bordeaux, 21/12/2015. 
Ces quatre témoignages montrent que la nature et la succession des états affectifs sont globalement semblables que le chercheur se trouve au Népal ou en France. La surprise surgit d'abord, à la suite de la perturbation de son environnement. Elle s'accompagne d'un sentiment immédiat d'effroi pour celui qui vit physiquement l'événement dans son corps avec la peur de mourir seul et comme réaction immédiate la fuite, et pour les autres d'un instant de sidération voire de déni. L'arrivée des premières images impose alors brutalement la réalité du séisme à ceux restés en France. L'anxiété produite par l'incertitude quant au sort des proches et à l'ampleur spatiale de la catastrophe s'accroît alors pour VAL et IS avec l'attente de nouvelles d'EJ et d'amis népalais. II est à noter que cet état d'anxiété est commun à tous, quelle que soit la position occupée par chacun. Sans liaison téléphonique, EJ ne peut appréhender la gravité du séisme qu'à l'échelle du lieu où il se trouve et d'une métrique uniquement pédestre. L'absence de visibilité renforce son angoisse et son sentiment $d$ 'isolement qu'il comble en se rapprochant d'autres individus. Pour ceux qui sont en France et qui peuvent communiquer entre eux, la mise en visibilité de l'évènement passe par les médias et s'effectue à une autre échelle, celle du Népal et de quelques régions emblématiques, d'où arrivent les premières images. Mais ces informations partielles et spatialement fragmentées laissent l'imagination combler les blancs, ce qui alimente tout autant leur angoisse. L'ignorance de la situation nourrit d'abord cette première phase émotionnelle. Puis, l'empathie et la tristesse apparaissent dans un second temps, au fur et à mesure que les informations se précisent. S'y ajoutent pour EJ et MF la crainte de la destruction supposée d'un terrain de recherche et la peur d'une remise en question de leur travail et de leur avenir. Dans tous les cas les émotions ont modifié notre perception du temps et de l'espace, nous donnant le sentiment d'être hors du monde, ici et ailleurs, dans un temps dilaté qui ne reprend sa forme qu'une fois les émotions retombées.

Les réponses de chacun aux émotions se différencient cependant dans leur nature comme dans leur temporalité. La nécessité pour EJ d'assurer sa survie ne lui permet pas de se laisser submerger par ses émotions. II en est de même pour VAL dont l'inquiétude par rapport au sort d'EJ occulte toute autre émotion se rapportant au Népal et à ses habitants. La mise en sécurité d'EJ prime avant tout et la peur commande l'action, qui consiste en sa mise à l'abri. Après le soulagement et la joie de le savoir vivant, il s'agit d'organiser son rapatriement. Une fois cette phase engagée, une seconde phase s'ouvre qui consiste pour MF et IS à activer les réseaux sociaux sur internet en quête d'autres amis népalais et en particulier de notre informateur de terrain, dont on est sans nouvelles. Le même choc émotionnel va ainsi conduire à des mises en action différenciées avec un partage tacite des tâches : recherche et diffusion de l'information pour les unes, action de rapatriement pour l'autre. Enfin, à la forte individualisation et personnalisation de l'émotion ressentie par tous (inquiétude quant au sort d'EJ, choc émotionnel à l'annonce de la mort de Dile) succède une extension et une généralisation de l'émotion à mesure que l'on découvre la situation dramatique d'autres lieux du Népal. L'émotion ne se focalise plus sur un seul lieu mais sur plusieurs. Les victimes ne sont plus des individus connus et nommés, mais plus globalement des habitants de tels ou tels village ou région du Népal. Les réseaux sociaux, qui mettent en relation $d^{\prime}$ 'innombrables individus originaires du monde entier et dont le point commun est d'être tous reliés émotionnellement à l'événement séisme, amplifient alors les émotions ressenties individuellement par le seul fait de les partager collectivement par les messages et les photographies postées. Comme le rappelle Durkheim, il existe une contagion de l'émotion liée aux interactions sociales: "les sentiments humains s'intensifient quand ils s'affirment 
collectivement. La tristesse comme la joie s'exalte, s'amplifie en se répercutant de conscience en conscience » (2003:572).

Toutefois, si les émotions ressenties sont communes à tous, leurs effets, comme on vient de le voir, se différencient à l'échelle des individus: cette différenciation renvoie à des systèmes de référentialités propres à chaque chercheur. Les émotions ici analysées convoquent ainsi trois grands systèmes de référentialités. Le premier est celui de la relation au terrain. Le deuxième est celui qui a trait à des événements passés de nature similaire réactivé et réactualisé par le séisme. Le troisième renvoie au statut de chacun des membres de l'équipe : étudiant ou enseignant-chercheur.

\section{Emotion et systèmes de référentialités : du rapport émotionnel au terrain}

Le rapport du chercheur au terrain, les représentations qu'il en a, ses expériences et son vécu, sont essentiels dans la façon dont se sont exprimées ces émotions. L'approche critique du statut du terrain en géographie et son renouveau épistémologique favorisent la reconnaissance de sa dimension subjective (L'Information Géographique, 2010/1; Les Annales de Géographie, 2012/5-6). Au-delà d'un simple rapport d'enquêteur/enquêtés, les chercheurs " habitent " leur terrain (Retaillé, 2010) et peuvent tisser des liens d'affect avec les habitants, négatifs ou positifs, le terrain étant d'abord une rencontre (Collignon, 2010). Chez l'habitant, les chercheurs ont passé du temps, vécu des moments dans des familles, dans des lodges, dans les villages. Ils ont souvent partagés des émotions avec leurs interlocuteurs, que ce soit dans le cadre de méthodes d'observation participante (Fernandez, 2005) ou en marge de ces recherches, dans ces "à-côtés " qui font toute la richesse d'un terrain. Ils ont été accompagnés par des informateurs ou traducteurs qui les ont assistés dans leurs travaux et avec qui ils ont noués des liens souvent privilégiés qui dépassent parfois le cadre d'une simple relation professionnelle. Cependant, au-delà de l'espace référentiel commun à l'équipe qu'est le terrain du Khumbu, les émotions ressenties se différencient par le statut occupé par chacun et par la convocation d'autres espaces de référence et d'évènements passés qui font écho au présent.

Pour EJ et MF, le Khumbu constitue un terrain de recherche neuf, sans antériorité, vécu au présent, celui de leur master et de leur thèse, qui participe à construire leur avenir professionnel et dont le séisme entrave la réalisation. Pour EJ, les effets de la première secousse l'oblige à reconstruire son espace de référence, à réactualiser sa carte mentale du Khumbu au prisme de ses connaissances de géographe, pour pouvoir se projeter dans un avenir immédiat : celui de sa mise en sécurité d'abord, celui de la poursuite d'un terrain de thèse bouleversé aussi bien physiquement qu'émotionnellement par le séisme ensuite. Serat-il capable d'y retourner sans revivre ce traumatisme ? Pourra-t-il y retrouver ce qu'il y a observé et vécu auparavant ? Pour MF, s'exprime l'angoisse de son incapacité à écrire sur un terrain qui n'existe peut-être plus, alors qu'il a été le révélateur de son goût pour la recherche.

Dans les récits d'EJ et MF l'anxiété prime quant à l'avenir d'un terrain déterminant pour la validation de leur diplôme. Elle s'explique par leur statut d'étudiant et leur place dans le programme. Comment vont-ils achever leur travail ? Quelle validité aura-t-il dans le contexte post-séisme ? À cela s'ajoute la culpabilité de penser à ce terrain, d'abord en tant qu'objet scientifique et non en tant que territoire habité par des personnes qu'ils ont fréquentées 
quotidiennement. Cependant, la conviction d'être les dépositaires des données recueillies auprès des populations avec la perspective d'une restitution future des résultats auprès de ces dernières contrebalance ce sentiment.

À la différence d'EJ et de MF, VAL et IS ont une relation différente au terrain qui est liée à leur expérience et à leur statut au sein du programme: ils les conduisent à se sentir responsables d'un doctorant, d'une équipe et d'un programme. La première se focalise ainsi sur le rapatriement d'EJ, une priorité vécue comme l'unique condition d'un retour à la " normale ». La seconde s'attache d'abord à identifier et localiser les membres de son équipe avant de se consacrer totalement à la recherche de ses connaissances et amis népalais. Mais surtout, les émotions de VAL et d'IS ressenties lors du séisme se différencient de celles des plus jeunes car elles font écho à des émotions vécues sur d'autres terrains, au Népal mais aussi ailleurs, sur des terrains qui appartiennent au passé, mais dont l'événement séisme ranime la mémoire d'événements douloureux (Beaude, 2012). Pour IS, la disparition de Dilé réveille l'émotion d'une autre mort, $d$ 'un guide encore plus proche, dans une avalanche, au Népal. La nouvelle de la localisation de l'épicentre lui fait brutalement prendre conscience que son ancien de terrain de thèse, auquel elle était fortement attachée, a été rayé de la carte. C'est tout un pan de son passé qui disparaît. Ces espaces de références, ont été fondateurs non seulement d'une carrière mais aussi d'une construction de soi (Retaillé, 2010), ce qui amplifie les émotions ressenties. Pour VAL, le séisme réactive l'émotion d'un autre séisme (Haïti, 2010), d'une autre doctorante, soumise à un danger similaire mais aussi d'évènements passés sur son propre terrain de thèse. Sa peur d'alors, de mourir à une place dont on ne comprend pas pourquoi on l'occupe, toujours subaffleurante, la projette dans la solitude et l'incompréhension qu'elle suppose qu'EJ est en train de vivre, au regard de sa propre expérience. L'émotion suscitée par le séisme fait ainsi surgir à la façon d'un boomerang des interactions spatiales jusque là insoupçonnées entre le terrain du Khumbu, et d'autres terrains situés à l'intérieur ou hors du Népal (Haïti, Guinée), qui passent par l'individu et son ressenti. En effet, "à côté du critère spatial et du critère scalaire, le critère temporel est également à considérer, combiné ou non aux précédents. Ainsi l'espace de référence peut-il appartenir au passé, qu'il s'agisse du passé local ou du passé d'un autre lieu. Un cas particulier est représenté par la référence qu'un espace fait à son propre passé, en utilisant ses "mémoires", pour employer le terme de F. Durand-Dastès " (Djament, 2005 : 11). L'émotion fonctionnerait ainsi comme l'actant d'une interspatialité qui ne se manifesterait que dans ce contexte précis.

L'expérience émotionnelle vécue par les auteurs à la suite du séisme confirme en tous cas que le terrain n'est en aucun cas une extériorité, indépendante du chercheur (Retaillé, 2010), mais qu'elle participe de son éthique et de son être au monde. L'émotion provoquée par l'événement séisme réactive d'autres émotions antérieures qui rappellent à chacun la dimension incontournable mais le plus souvent refoulée du sensible dans la recherche. La familiarité avec certains terrains, entretenue par des séjours longs ou répétés, les inscrit de fait dans les lieux et espaces de l'habiter du chercheur, autrement dit dans la sphère de son vécu le plus intime. Elle favorise ainsi une certaine forme d'identification et d'empathie dès que ce terrain ou des proches sont touchés par un événement tragique. Mais l'expérience émotionnelle du séisme ne met pas seulement en lumière le lien entre émotions et statut du terrain. Elle révèle aussi différentes formes d'interspatialités que suscite " ici » l'émotion liée à un événement survenu "là-bas ", en interrogeant notre rapport à la distance et notre 
définition du proche et du lointain dans notre façon de ressentir et de gérer nos émotions et dans ce que ces dernières produisent en retour en terme d'action.

\section{Appréhender l'espace et la distance ou comment gérer ses émotions pour agir : un jeu de places et de placements}

Le but de cette dernière partie est de comprendre comment différents états affectifs, plus particulièrement l'effroi, l'anxiété et la colère, influent sur la relation que les chercheurs entretiennent avec l'espace, dans une dimension d'interface, d'emboîtement ou de cospatialité, et de comprendre comment en retour la façon dont les chercheurs appréhendent et maîtrisent l'espace permet de gérer l'émotion : par des stratégies de mise à l'abri du danger, par l'écriture, par le recours aux réseaux sociaux, par le déploiement de tous les moyens pour faire rentrer EJ.

Email d'EJ à VAL et IS, le 27 avril 2015 à 7h00

[...] Il y a eu quelques secousses dans la nuit. On a dormi tout habillé et à l'extérieur du duvet pour pouvoir s'enfuir en cas de danger. Nous sommes repartis le lendemain - hier - à Namche sachant qu'une autre secousse allait se produire. On pensait se mettre à l'abri sur le replat de Khumjung mais visiblement toutes les maisons se sont écroulées. A midi une nouvelle secousse (5-6 ?) a eu lieu à Namche, encore une fois nous avons pu contrôler. La grande majorité des habitants et propriétaires de lodges de Namche sont partis dans l'après-midi sur les hauteurs pour passer la nuit (plus par superstition que par sécurité ?). Nous n'avions pas de tentes, nous sommes restés à Namche (très peu affecté) avec quelques touristes. L'atmosphère était incroyable. Nous avons acheté des provisions, un réchaud et avons squatté un lodge avec deux Français. [...] Je vous tiens au courant.

Durant les jours qui ont suivis la première secousse, les actions d'EJ sont sous-tendues par un jeu de (dé)placement. "Toujours intervient, au moment des choix, l'activité de discrimination du proche et du lointain et, surtout, de ce qui est acceptable en la matière. De qui, de quoi accepté-je ou recherché-je la proximité, de qui, de quoi dois-je m'éloigner, me distancier, voire, en sus, me protéger? " (Lussault, 2009 : 79). Dans le contexte du séisme, ce jeu de placement est d'abord motivé par la quête du meilleur positionnement possible, qui s'exprime par une distanciation vis-à-vis des lieux de dangers. Lors de la première secousse, l'effroi s'associe à une réaction instinctive de fuite qui vise à atteindre une position estimée moins risquée. Puis le soulagement d'être encore en vie laisse vite place à une inquiétude liée à l'absence de visibilité : invisibilité de son environnement immédiat (brouillard), manque de visibilité concernant l'ampleur et la gravité de la situation (interruption de toutes télécommunications durant les premières heures). C'est l'utilisation d'un téléphone satellite qui le reconnecte au reste du monde qui lui redonne de la visibilité sur son environnement et le place dans un rapport d'emboîtement ${ }^{2}$ pour accéder au lointain comme au proche. Dès lors, l'accès à des moyens de télécommunication conditionne le rapport à l'espace du " rescapé » et ses stratégies d'action. Le choix du lieu où attendre le rapatriement résulte d'un arbitrage entre des paramètres de sécurité et de "joignabilité » (Sauvageot, 2003).

2 L'emboîtement est l'une des trois familles de l'interspatialité, il représente " l'inclusion d'espaces les uns dans les autres [et] relie les espaces par une transformation scalaire (petit/grand) et par une inclusion de l'un dans l'autre. » (Lévy \& Lussault, 2003 :306). 
Namche Bazar dispose de replats sécurisés au-dessus des maisons et d'un système de communications fonctionnel. Mais en l'absence de tentes et de places disponibles, EJ privilégie Khumjung et ses baraques en bois malgré son accès plus restreint aux télécommunications.

Dans les premières heures qui succèdent au séisme, les chercheurs en France font de leur côté l'expérience de la séparation, qui en les éloignant d'êtres ou de choses qui comptent pour eux, les projettent dans une situation d'insécurité, d'anxiété, voire d'agressivité (Sillamy, 1989: 252). L'impossibilité de communiquer avec le Népal les renvoie à une incapacité d'agir et déclenche un sentiment d'impuissance. La distance métrique FranceNépal est irréductible, infranchissable, seuls leurs imaginaires, fortement alimentés par les images médiatiques, leur permettent d'envisager cet ailleurs injoignable. Ces images, souvent répétitives mais de plus en plus nombreuses ne fournissent dans un premier temps aucune visibilité de l'environnement d'EJ.

Comment agir sur cet espace lointain depuis notre propre place ? " Maîtriser la distance qui nous sépare de l'ailleurs, c'est nous donner les moyens de maîtriser notre existence et notre devenir, c'est nous donner les moyens de conformer nos intentions et nos actions " (Beaude, 2012 : 8). C'est aussi l'unique moyen pour VAL, IS et MF d'obtenir des nouvelles de leurs connaissances, de protéger et de faire revenir EJ, d'envisager un " après ". Astreintes à une fixité ou tout au moins à une immobilité relative, composer avec la distance et agir ne deviennent envisageables que par la possibilité d'installer une "coprésence médiée " ${ }^{3}$, une cospatialité, soit "la possibilité d'embrayer une action (fût-ce un simple acte de langage) dans un espace à partir d'un autre, via les réseaux télécommunicationnels qui assurent la médiation entre des points éloignés " (Lussault, 2009 : 79). L'action est tributaire de la possibilité technique d'établir une proximité topologique. Pour IS et MF ce processus se traduit, par un recours presque frénétique aux médias et aux réseaux sociaux qui les projettent mentalement au Népal pour y chercher de l'information et la diffuser. L'hyperspatialité, qui les connecte à des inconnus situés dans d'autres pays et concernés par les événements, constitue un puissant relais des émotions vécues au Népal mais aussi dans le reste du monde. Elle transforme les auteurs immobilisés en France derrière leurs écrans, en commutateurs spatiaux (Lussault, 2014). Pour VAL, l'objectif plus ciblé, est conditionné par la mise en action d'un acteur extérieur à l'équipe de recherche en la personne de l'assureur. Ce médiateur, localisé dans un bureau parisien, n'entretient a priori aucun état affectif ni avec le terrain, ni avec EJ mais occupe une place fondamentale dans le dispositif de rapatriement. Lui seul peut régler le problème de distance métrique entre le village de Khumjung, où se trouve EJ, l'altiport régional de Lukla et l'aéroport international de Katmandou à partir duquel il pourra rejoindre la France. Mais l'événement du $1^{\mathrm{er}}$ mai (récit VAL) montre à quel point l'assureur, qui n'entretient de lien avec l'événement qu'au travers d'un dossier administratif, n'a qu'une représentation extrêmement partielle de la situation. Celle-ci s'avère impuissante à produire une action de cospatialité efficace. Ce régime de gestion de la distance, considéré comme inacceptable par VAL, provoque chez elle une agressivité à l'encontre de l'assureur, cristallisation du retour d'une émotion, jusque là soigneusement mise à distance. L'activation de ces différentes cospatialités a fini par

\footnotetext{
${ }^{3}$ Internet, pour B. Beaude (2012), constitue un lieu de synchoristation, c'est à dire, le « processus qui consiste à se donner un espace commun pour être et pour agir », qui permet la mise en relation des deux réalités, d'une spatialité complémentaire.
} 
produire l'effet attendu : le franchissement de la distance de l'espace euclidien et le retour d'EJ sur le sol français.

\section{Conclusion : événement catastrophique, émotion, distance et éthique}

Le surgissement de l'émotion dans le contexte du séisme rappelle toute la complexité de la relation d'un chercheur à " son " terrain, des mémoires qu'il porte, des liens affectifs qui s'y nouent, surtout lorsque celui-ci a été bouleversé de façon brutale. Si tout un chacun peut ressentir de l'émotion face à une catastrophe, même si elle ne nous touche pas directement, l'intensité de cette émotion est largement fonction de la relation particulière et affective que nous tissons avec les personnes ou les lieux concernés. L'attachement au terrain, autrement dit sa proximité, est d'autant plus grand qu'il a participé à notre construction scientifique et à notre reconnaissance en tant que chercheur. Les émotions vécues lors du séisme soulignent le poids de l'implication interactionnelle et intersubjective dans la façon de travailler son terrain et conduit à réfléchir à la place que l'on est prêt à leur donner dans un travail de recherche (voire dans notre existence), des manières de les mettre à distance ou de les mobiliser en vue de l'action.

Le bouleversement occasionné par une perturbation extérieure pose en effet à chacun des questions éthiques fondamentales: au-delà des émotions ressenties, quel retour sur le terrain au sens propre - revenir sur les lieux de la catastrophe - comme au sens figuré quelle restitution du travail de terrain ante-séisme effectuer auprès des populations? Doit on oublier/refouler ses émotions et se concentrer sur d'autres terrains moins chargés d'affect ? Peut-on, doit-on y revenir comme si rien ne s'y était passé, indifférent au malheur d'autrui, en se réfugiant derrière son statut de chercheur et sa prétendue objectivité ? Ou bien doit-on assumer la dimension émotionnelle de toute recherche, tout en dépassant l'événement circonstanciel qui l'a exacerbé, par un travail réflexif plus approfondi ? Dans ce cas, jusqu'à quel point s'extimiser sans tomber dans une égo-géographie trop nombriliste ? Comment même rendre compte des émotions dès lors que l'écriture a posteriori les met déjà à distance ? Faut-il faire de l'émotion un ressort de l'action en s'engageant dans une géographie impliquée et dans des actions d'aide individuelles ou collectives au risque de mettre en péril la recherche et d'induire des effets pervers, des formes d'instrumentalisation sur son terrain ? Chacun répondra à son niveau individuel à ces redoutables questions. La communauté de chercheurs français sur le Népal y a, quant à elle, répondu à sa manière en créant un réseau de solidarité, le Réseau Chercheurs Népal, fort de 70 chercheurs et étudiants, afin de partager son expertise sur les sociétés et les territoires avec les ONG et les associations travaillant sur le terrain ${ }^{4}$.

Enfin, bien que nous vivions dans un monde hyperconnecté, où médias et réseaux sociaux permettent la cospatialité avec des évènements géographiques localisés, l'événement du 25 avril 2015 a également rappelé de manière violente aux quatre auteurs géographes, le caractère irréductible de la distance euclidienne, au-delà de toutes les stratégies qu'ils ont pu déployer pour tenter de la réduire. Les émotions provoquées par cet événement lointain ont envahi notre espace du quotidien, notre espace privé. Vécues et amplifiées selon le

\footnotetext{
${ }^{4}$ Le réseau s'est engagé à aider à la reconstruction du village de Salme, dans le Centre-ouest du Népal détruit à $80 \%$ par le séisme, où de nombreux chercheurs, stagiaires et étudiants du CNRS et de l'INRA se sont succédés entre les années 1980 et 2000.
} 
système de référentialités propre à chacun et selon les places occupées, elles ont néanmoins contribué à nous rapprocher les uns des autres et à construire un nouveau système de référentialité partagé, qui reconfigure en retour notre rapport au terrain et participe plus généralement à la construction de notre rapport au monde.

\section{Bibliographie}

BEAUDE B. (2012), Internet, changer l'espace, changer la société, Editions Fyp, http://www.beaude.net/icecs/, consulté le 14/01/2015.

BLOCH H., VURPILLOT E. (1994), Grand dictionnaire de la psychologie, Paris, Larousse, 864 p. CHARRON C., et al. (2014), Les 500 mots de la psychologie, Paris, Dunod, 331 p.

CLAVAL P. (1996), La géographie comme genre de vie: un itinéraire intellectuel, Paris, L'Harmattan, $144 \mathrm{p}$.

COLLIGNON B. (2010), "L'éthique et le terrain ", L'information géographique, n²010/1, pp. 63-83, en ligne : http://www.cairn.info/revue-l-information-geographique-2010-1-page63.htm

DJAMENT G. (2005), "Pour une géographie de l'interspatialité. L'exemple romain », $7^{\text {èmes }}$ Rencontres Théo Quant, janvier 2005, 13 p., en ligne: http://thema.univfcomte.fr/theoq/pdf/2005/TQ2005\%20ARTICLE\%2016.pdf

DURKHEIM E. (2003), Les formes élémentaires de la vie religieuse, le système totémique en Australie, Paris, PUF, $1^{\mathrm{e}}$ éd. 2012, 672 p.

FERNANDEZ F. (2005), "L'engagement émotionnel durant l'enquête sociologique : retour sur une observation " anonyme " auprès d'ex usagers des drogues ", Carnets de bords de la recherche en sciences humaines, $n^{\circ} 9$, pp. 78-87, en ligne: https://hal.archivesouvertes.fr/hal-00488078/document

LABUSSIERE O., ALDHUY J. (2012), " Le terrain, c'est ce qui résiste. Réflexion sur la portée cognitive de l'expérience sensible en géographie ", Annales de géographie, n²015/5, pp. 583-599, en ligne: https://www.cairn.info/revue-annales-de-geographie-2012-5-page583.htm

LUSSAULT M. (2009), De la lutte des classes à la lutte des places, Paris, Grasset, 221 p.

RETAILLE D. (2010), "Au terrain, un apprentissage ", L'information géographique, n²010/1, pp. 84-96, en ligne : https://www.cairn.info/revue-l-information-geographique-2010-1-page84.htm

RIME B. (2005), Le partage social des émotions, Paris, PUF, 419 p.

SAUVAGEOT A. (2003), L'épreuve des sens, de l'action sociale à la réalité virtuelle, Paris, Presses Universitaires de France, $256 \mathrm{p}$.

SILLAMY N. (1989), Dictionnaire de la psychologie, Paris, Larousse, 290 p.

VOLVEY A., CALBERAC Y., HOUSSAYE-HOLZSCHUCH M. (2012), "Terrains de je. (Du) sujet (au) géographique ", Annales de géographie, $n^{\circ} 5 / 2012, n^{\circ} 287-288, p p .441-461$, en ligne : http://www.cairn.info/revue-annales-de-geographie-2012-5-page-441.htm 